\title{
Late-Onset Pompe Disease With Left-Sided Bronchomalacia
}

\author{
Chia-Feng Yang MD, Dau-Ming Niu MD PhD, Mei-Jy Jeng MD PhD, \\ Yu-Sheng Lee MD, Pei-Chen Taso MD, and Wen-Jue Soong MD
}

\begin{abstract}
Pompe disease is a rare autosomal recessive disorder caused by $\alpha$-glucosidase deficiency. Lower airway involvement and management are rare in patients with late-onset Pompe disease. We describe the case of a 16-y-old girl with late-onset Pompe disease who presented with obvious progressive deterioration in respiratory function. Pulmonary hypertension was also apparent on echocardiography. She had been on enzyme replacement therapy and nighttime CPAP ventilation for several years. Flexible bronchoscopy was used for diagnosis and subsequent implantation of a bronchial airway stent. Following implantation of the stent, the patient's pulmonary function stabilized, and her pulmonary hypertension resolved. The patient continued on enzyme replacement therapy and nighttime CPAP ventilation. This case highlights that lower airway involvement may occur with lateonset Pompe disease and that flexible bronchoscopy can be an effective tool for both diagnosis and management of lower airway collapse in late-onset Pompe disease. Key words: bronchomalacia; bronchial stent; flexible bronchoscopy; late-onset Pompe disease. [Respir Care 2015;60(2):e26-e29. (C) 2015 Daedalus Enterprises]
\end{abstract}

\section{Introduction}

Pompe disease, also known as glycogen storage disease type II or acid maltase deficiency, is a very rare ( 1 in 40,000-600,000) autosomal recessive disorder caused by acid $\alpha$-glucosidase deficiency and consequent accumulation of glycogen in lysosomes and cytoplasm. ${ }^{1,2}$ This accumulation results in cell damage and has various clinical manifestations depending on the timing of onset. The most severe form of Pompe disease is infantile-onset Pompe disease, which becomes apparent within the first 2 months of life and is characterized by cardiac hypertrophy, hypotonia, respiratory distress, and delayed motor development. ${ }^{3,4}$ Late-onset Pompe disease may become evident at

The authors are affiliated with the Department of Pediatrics, Taipei Veterans General Hospital, Taipei, Taiwan. Drs Niu and Soong are also affiliated with the Department of Pediatrics, School of Medicine, National Yang-Ming University, Taipei, Taiwan.

The authors have disclosed no conflicts of interest.

Correspondence: Wen-Jue Soong MD, National Yang-Ming University, No. 155, Section 2, Linong St, Taipei, 112 Taiwan, Republic of China. E-mail: wjsoong@vghtpe.gov.tw.

DOI: $10.4187 /$ respcare.03419 any age and is typically characterized by myopathy, respiratory insufficiency, and obstructive sleep apnea. ${ }^{5-8}$ The myocardium is usually not affected in late-onset Pompe disease; however, cor pulmonale may occur. Regardless of the timing of onset, enzyme replacement therapy with recombinant human acid $\alpha$-glucosidase is recommended. ${ }^{1}$

The upper airway, inspiratory muscles, and diaphragm are frequently affected in late-onset Pompe disease, ${ }^{1}$ whereas, to the best of our knowledge, lower airway involvement has not been reported. Here, we present a unique case of late-onset Pompe disease with obvious left main bronchial malacia that was diagnosed and subsequently managed with stent implantation by flexible bronchoscopy.

\section{Case Report}

A 16-y-old girl presented with respiratory distress in September 2012. She had previously been diagnosed with late-onset Pompe disease (gene mutation c.2238G $>\mathrm{C}$ [p.W746C]/c.2815_2816del [p.V939LfsX78]) at $9 \mathrm{y}$ of age and had been receiving enzyme replacement therapy (20 mg/kg every 2 weeks) since October 2007. Pulmonary function testing over the following years revealed restrictive lung disease and progressively deteriorating lung function (Fig. 1); FVC and $\mathrm{FEV}_{1}$ had decreased by $>30 \%$ in a $3-y$ period. In 2009, the patient was diagnosed with 


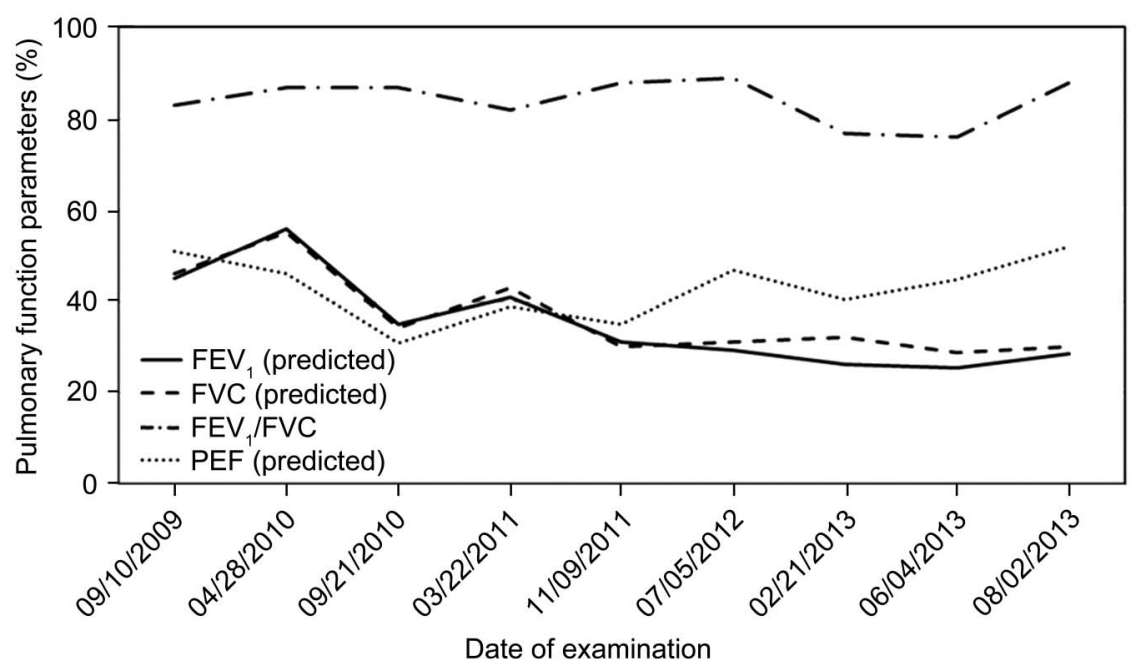

Fig. 1. Summary of the patient's pulmonary function parameters over an $\sim 4-y$ period. PEF $=$ peak expiratory flow.
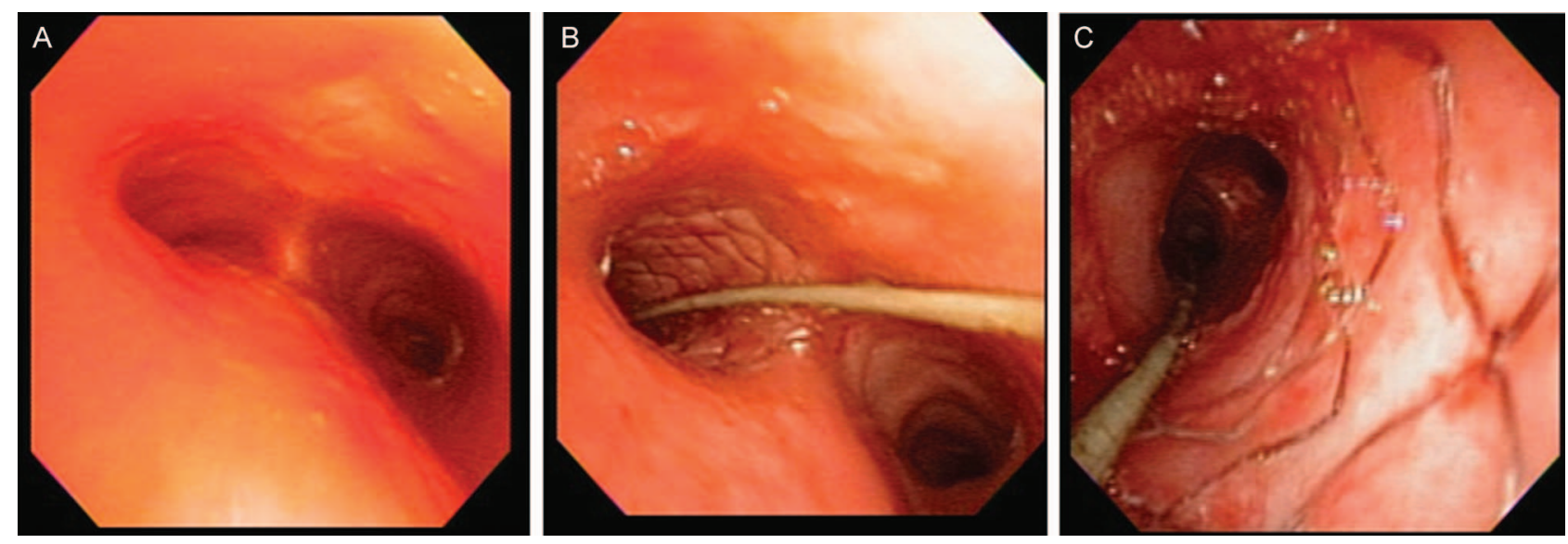

Fig. 2. Flexible bronchoscopy images taken before $(A)$ and after $(B$ and $C)$ implantation of a metallic mesh stent in the left main bronchial lumen.

obstructive sleep apnea syndrome by polysomnography and was prescribed CPAP therapy. Overnight polysomnography performed in December 2011 revealed 59 hypopneic events and 2 apneic events. Echocardiography performed in July 2012 demonstrated: mild mitral valve prolapse, thickening $(0.38 \mathrm{~cm})$, and regurgitation (grade $2+$ ); mild tricuspid valve prolapse and regurgitation (grade $2+)$; and mild pulmonary hypertension $(35 \mathrm{~mm} \mathrm{Hg}$ ), minimal pulmonary regurgitation, and inferior vena cava dilatation without collapse during inspiration.

Upon presentation in our hospital, the girl reported a recent history of sleep disturbance with difficult respiration. She also reported experiencing exercise dyspnea, chest pain, and palpitations. She had mild scoliosis. Laboratory results were as follows: creatinine, $0.33 \mathrm{mg} / \mathrm{dL}$; lactate dehydrogenase, $311 \mathrm{U} / \mathrm{L}$; alanine aminotransferase, $77 \mathrm{U} / \mathrm{L}$; aspartate aminotransferase, $109 \mathrm{U} / \mathrm{L}$; creatine kinase, 305 $\mathrm{U} / \mathrm{L}$; white blood cell count, 6,500 cells/ $\mu \mathrm{L}$; hemoglobin,
$137 \mathrm{~g} / \mathrm{L}$; and platelets, 180,000 cells/ $\mu \mathrm{L}$. She was admitted for respiratory examination and noninvasive ventilation. Flexible bronchoscopy under intravenous sedation revealed compromise of the pharynx, lower third tracheal malacia, and severe left main bronchial malacia with lumen collapse of $>90 \%$ (Fig. 2A). Chest computed tomography findings were consistent with flexible bronchoscopy findings (Fig. 3). Due to the progressive dyspnea, she was scheduled for aggressive management. A metallic expandable mesh stent $(9 \times 30 \mathrm{~mm})$ was implanted smoothly using a flexible bronchoscope (Figs. 2B and 4). After stent implantation, the left main bronchial lumen became patent (Fig. 2C) and clear at the first bifurcation. Enzyme replacement therapy and nighttime CPAP were continued.

Follow-up echocardiography performed in August 2013 revealed no evidence of mitral valve prolapse or mitral or pulmonary regurgitation and minimal tricuspid regurgitation (grade 1-). Pulmonary pressure was $16-20 \mathrm{~mm} \mathrm{Hg}$. 


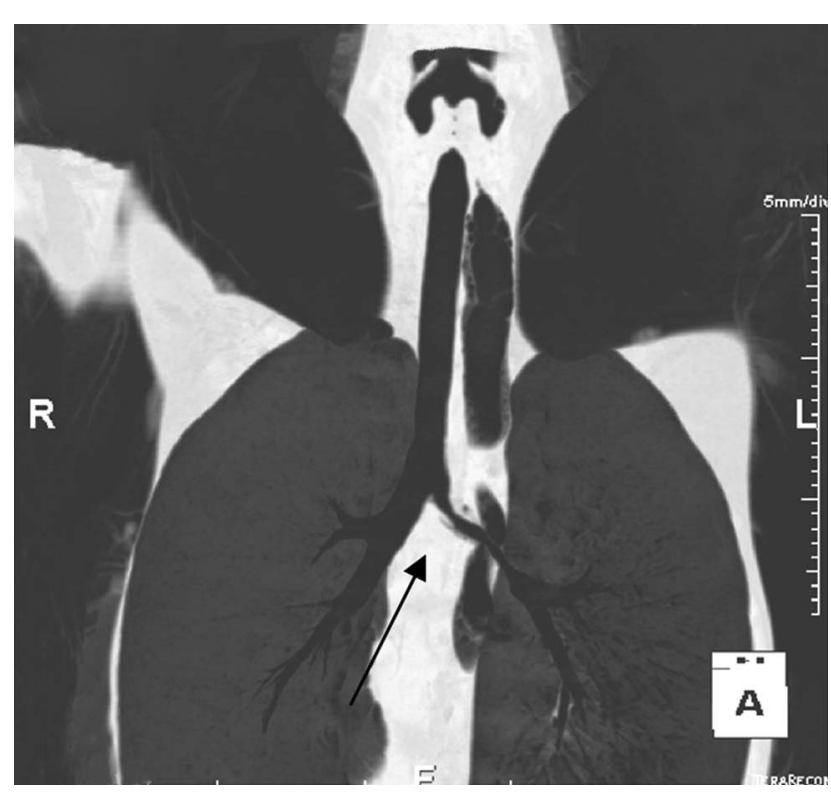

Fig. 3. Chest computed tomography image shows severe lumen narrowing of left main bronchus (arrow).

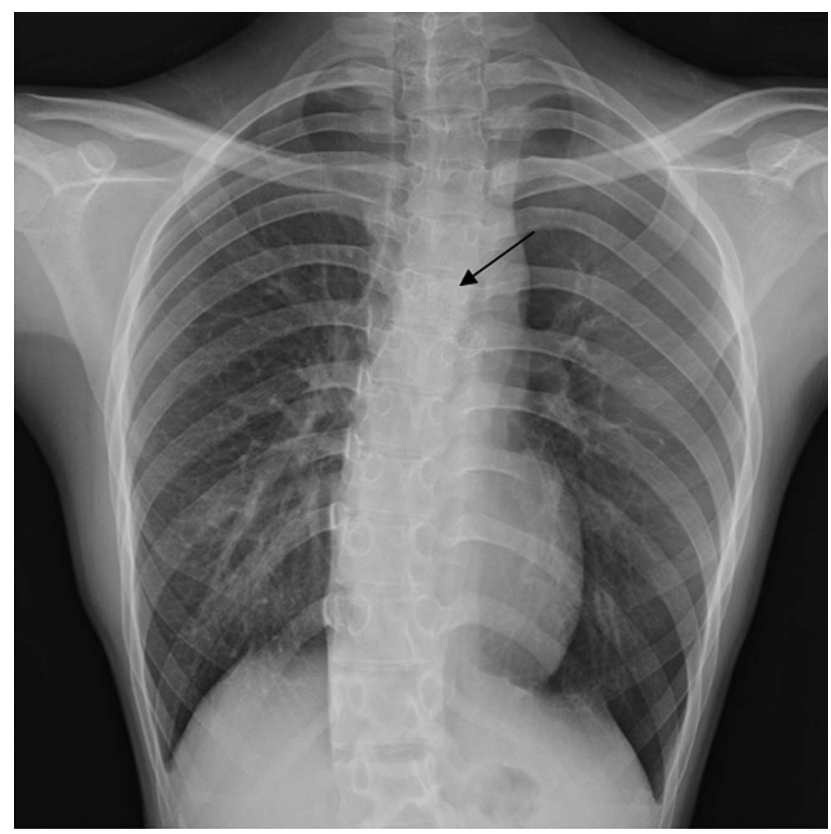

Fig. 4. Chest radiograph after implantation of a left main bronchial stent (arrow)

Treadmill exercise testing revealed an improvement from $259 \mathrm{~m}$ (before stent) to $302 \mathrm{~m}$ in $6 \mathrm{~min}$. Follow-up overnight polysomnography findings in August 2013 were consistent with a diagnosis of mild obstructive sleep apnea syndrome (39 hypopneic events and 4 apneic events). Notably, CPAP completely ameliorated the obstructive sleep apnea syndrome (one hypopneic event and zero apneic events). At the last flexible bronchoscopy examination
(8 months after stent implantation), the left main bronchial lumen and stent showed patency and no granulation formation. The patient was attending school on a daily basis, taking regular enzyme replacement therapy, and undergoing follow-up examinations. Her pulmonary function was stable, and the pulmonary hypertension had completely resolved.

\section{Discussion}

To our knowledge, this is the first reported case of late-onset Pompe disease with lower airway involvement. In addition to the atypical involvement of the lower airway, the patient's presentation was unusual in that she exhibited precipitously declining lung function even with ongoing enzyme replacement therapy, but did not appear to have deteriorating skeletal muscle strength.

A previous study carried out in The Netherlands by van der Beek et $\mathrm{al}^{9}$ showed that nearly three-quarters of subjects with late-onset Pompe disease had pulmonary dysfunction and that nearly $40 \%$ had clear respiratory muscle weakness. Mild scoliosis was also quite common among the 92 subjects included in the study. Disease duration, skeletal muscle weakness, and sex were all found to be predictors of pulmonary dysfunction, with males being more severely affected than females. Interestingly, despite the high level of respiratory involvement (nearly 30\% required some form of respiratory support), a high proportion $(\sim 40 \%)$ of the subjects had not undergone previous vital capacity testing, emphasizing that respiratory testing in patients with late-onset Pompe disease is underperformed. The study by van der Beek et al $^{9}$ highlights the key typical features of late-onset Pompe disease.

Clearly, any ongoing deterioration in respiratory function is concerning and necessitates the need for meticulous examination and appropriate intervention(s) to reverse or halt further progression. After admission, our patient underwent flexible bronchoscopy examination, which revealed not only upper airway compromise (common in patients with Pompe disease) but also significant lower airway narrowing with tracheomalacia and left main bronchial malacia. Flexible bronchoscopy was subsequently safely used for both bronchial stent implantation and follow-up airway examinations. If flexible bronchoscopy is used early, this management can detect not only respiratory lesions, if present, but also effectively halt further deterioration and facilitate recovery from complications, such as pulmonary hypertension and infection. This case leads us to suggest that a thorough airway examination is crucial for patients with late-onset Pompe disease who experience an unexpectedly fast progression of respiratory impairment. Indeed, regular flexible bronchoscopy examinations in these patients may be warranted to facilitate early recognition of any adverse changes. 


\section{Pompe Disease With Bronchomalacia}

There are several potential mechanisms underlying the lower airway involvement in our patient with late-onset Pompe disease. Clearly, disease-related weakening of the posterior tracheobronchial muscles leading to dynamic collapse is likely. As noted, our patient had mild scoliosis, which may have contributed to thoracic insufficiency and hence respiratory insufficiency. General respiratory muscle involvement is also likely.

The case described herein highlights that lower airway compromise may occur with late-onset Pompe disease and that flexible bronchoscopy can be an effective tool for both diagnosis and management of airway manifestations of late-onset Pompe disease. As mentioned previously, regular monitoring of respiratory function is important for patients with Pompe disease, ${ }^{9}$ regardless of enzyme replacement therapy. Appropriate airway support should be implemented as needed.

\section{REFERENCES}

1. Mellies U, Lofaso F. Pompe disease: a neuromuscular disease with respiratory muscle involvement. Respir Med 2009;103(4):477-484.

2. Pompe JC. [Concerning idiopathic hypertrophy of the heart]. Ned Tijdschr Geneeskd 1932;76:304-311. Article in Dutch.
3. van den Hout HM, Hop W, van Diggelen OP, Smeitink JA, Smit GP, Poll-The BT, et al. The natural course of infantile Pompe's disease: 20 original cases compared with 133 cases from the literature. Pediatrics 2003;112(2):332-340.

4. Kishnani PS, Hwu WL, Mandel H, Nicolino M, Yong F, Corzo D, et al. A retrospective, multinational, multicenter study on the natural history of infantile-onset Pompe disease. J Pediatr 2006;148(5):671676.

5. Müller-Felber W, Horvath R, Gempel K, Podskarbi T, Shin Y, Pongratz D, et al. Late onset Pompe disease: clinical and neurophysiological spectrum of 38 patients including long-term follow-up in 18 patients. Neuromuscul Disord 2007;17(9-10):698-706.

6. Wokke JH, Escolar DM, Pestronk A, Jaffe KM, Carter GT, van den Berg LH, et al. Clinical features of late-onset Pompe disease: a prospective cohort study. Muscle Nerve 2008;38(4):1236-1245.

7. Chien YH, Lee NC, Huang HJ, Thurberg BL, Tsai FJ, Hwu WL. Later-onset Pompe disease: early detection and early treatment initiation enabled by newborn screening. J Pediatr 2011;158(6):1023.e11027.e1.

8. Chien YH, Lee NC, Huang PH, Lee WT, Thurberg BL, Hwu WL. Early pathologic changes and responses to treatment in patients with later-onset Pompe disease. Pediatr Neurol 2012;46(3):168-171.

9. van der Beek NA, van Capelle CI, van der Velden-van Etten KI, Hop WC, van den Berg B, Reuser AJ, et al. Rate of progression and predictive factors for pulmonary outcome in children and adults with Pompe disease. Mol Genet Metab 2011;104(1-2):129-136. 